\title{
High Level Simulation \& Modeling for Medical Applications - Ultrasound Case
}

\author{
A. Chihoub \\ Siemens Corporate Research Inc., 755 College Road East, Princeton, NJ 08540, USA \\ chihoub@scr.siemens. com
}

\begin{abstract}
In this paper we will present the results of mapping and simulating the B-Mode (echo), and Doppler (flow) algorithms in ultrasound processing onto 1D and 2D based architectures. A scaleable parallel architecture using commercial off the shelf DSP processors was simulated/evaluated for performance and cost feasibility to replace an existing dedicated hardware solution. The results showed that such an architecture was both feasible and cost effective.
\end{abstract}

\section{Introduction}

Ultrasound processing generally has very high computational requirements. Developing the hardware necessary to meet such requirements, in real-time, can be quite expensive. Until recently, meeting such demands required the development of custom designed hardware.

Recent advances in processor technology combined with the drop in the price of digital signal processors (DSPs) have made it possible to develop cost efficient parallel processing systems using off the shelf commercial processors for use in ultrasound applications.

In this paper we will present the mapping of the algorithms used to perform BMode (echo) and Doppler (flow) processing in a typical ultrasound machine onto a linear, and a 2D mesh array of Digital Signal processors. We will also present the simulation results from running the parallelized algorithms on the target architectures using a high level simulation tool. The goal is to verify the functionality and extract the performance measures of the parallelized algorithms on the target architectures.

The rest of the paper is divided into the following sections. In the next section we will briefly describe the algorithms used in B-Mode, and Doppler processing in a typical ultrasound machine. In section three we will describe the mapping of the algorithms to the linear, and 2D mesh arrays. In section four we will briefly describe the SES/Workbench simulation model we used to validate the approach. In section five we will present the results of the simulation. Finally, in section six we will give some concluding remarks.

\section{Overview of Ultrasound Processing}

In this section we will give a brief overview of ultrasound processing and a summary of the processing steps encountered in B-Mode, and Doppler processing. The over- 
view is included to help the reader understand better the algorithm mapping discussions given in the next section. Figure 1 gives the flow diagram of the overall process. More details about ultrasound processing can be found in [1],[2] and [3].

Ultrasound data is typically gathered by sending acoustic pulses along some selected directions. The reflected pulses are then received and conditioned into broadband RF beam vectors for further processing. Once the RF vectors are formed they are passed through the digital receivers to perform the coherent down conversion and decimation filteration steps. The coherent down conversion step is used to downconvert the RF vectors into baseband signals each with an in-phase and a quadrature component. The decimation filteration step is then used to independently low pass filter, and sub-sample the in-phase and quadrature components of each signal. The low pass filtering step is used to remove the un-wanted frequency components generated by the coherent down conversion step. The sub-sampling step is used to reduce the size of each beam vector to the desired number of samples.

Once the vectors pass through the digital receivers they are routed to the proper processing segment to perform the processing steps dictated by the selected mode of operation of the ultrasound machine. The major steps in typical B-Mode, and Doppler processing pipelines are described next.

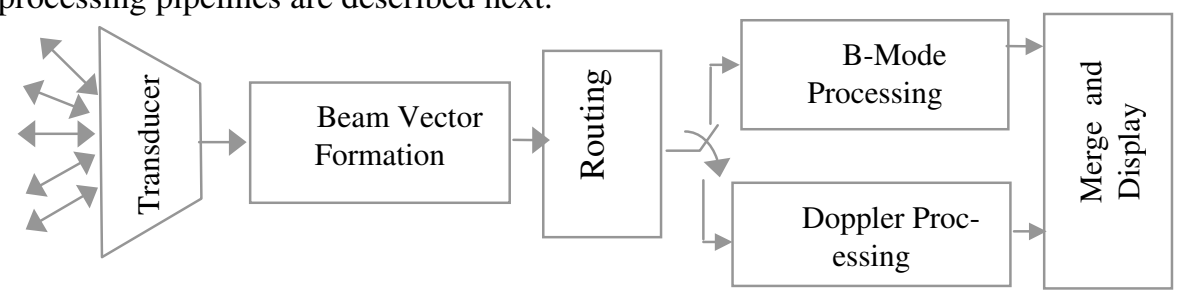

Fig. 1. Overview of ultrasound processing

\subsection{B-Mode Processing}

The first step in our B-Mode processing pipeline is magnitude calculation. In this step the filtered in-phase and quadrature $(I, Q)$ components of the echo signal are used to calculate the magnitude of each vector for all samples.

The next step is focal zone blending. In this step data from several beam vectors focused at different depths along the same direction are combined together into a single vector to increase the spatial resolution. The focal zone blending is followed with a look-up table step. This step compresses the dynamic range of the data using a logarithmic conversion function. An FIR filter with up to sixteen taps is then used to enhance the data along the axial (beam) direction in a step called axial edge enhancement.

In systems with parallel beam formation features, the RF data may be down converted with several different frequencies. The resulting vectors have the same direction but different, possibly overlapping, frequency bands. In such systems a step, called frequency compounding, is used to sum up these sets of vectors into a single vector. The frequency compounding step is followed by the dynamic reject step to remove low level signals. A threshold value calculated using a neighborhood of samples around the sample of interest is used to accept or reject the sample. If a sample is 
rejected its new value is set to zero. A three tap FIR filter is then used to provide additional edge enhancement in the lateral direction. The step used to perform this function is called lateral edge enhancement.

The next step, black hole filing, is used to compensate for the null regions caused by the coherent addition of individual scatterers which appear as black spots (holes) in the image. The black hole filing step calculates a threshold for a given sample based on its two nearest neighbors in the lateral direction. If the difference between the sample's value and either of its neighbors is less or equal to the threshold, the sample is passed unchanged to the next processing stage. Otherwise, its new value is calculated as the average between its original value and that of one of its neighbors. The black hole filing step is followed by the variable persistence step. The variable persistence step applies an IIR filter between succeeding frames to determine how much the values in the current frame are allowed to decay with respect to the previous frame. A user selected persistence and dynamic range values are used to determine the filter coefficients. Finally, another look-up table which implements logarithmic conversion to further compress the dynamic range of the data is used. The resulting data is passed to the scan converter for display. This step is not part of the echo processor and is not discussed here.

\subsection{Doppler Processing}

The first step in our Doppler processing pipeline is corner turning. In this step the I and Q flow vectors are re-arranged for ease of processing in later steps. The vectors come out of the digital receivers in depth order, the corner turning step re-arranges them in time order. This step does not require any explicit processing. It can be achieved by writing and reading the data into memory in a proper sequence.

The adaptive wall filter step is used to remove the clutter component of the Doppler signal (Doppler signal due to the probe and tissue movement) while keeping the Doppler signal due to the flowing blood. This step can be done by passing the time ordered I and Q vectors through a complex mixer so that the clutter component of the signal falls within the notch of two FIR filters used to remove the clutter from the I and $\mathrm{Q}$ vectors. The filtered I and $\mathrm{Q}$ may then be shifted back to the original frequency by passing them through the conjugate of the complex mixer used to do the original shift. This shifting step is application dependent and may be skipped. The frequency location of the clutter signal is calculated by the clutter estimator. The calculated estimate is then used to select the coefficients of the FIR filters used to remove the clutter. The FIR filters may operate in one of three modes: Linear Time Invariant (LTI) mode, Circular mode or Linear Time Varying (LTV) mode.

Once the wall filter step is completed the filtered vectors of each ensemble are used to calculate a velocity estimate using autocorrelation. For each flow ensemble a triplet of the power estimate of the signal $\mathrm{P}(\mathrm{n})$, the numerator $\mathrm{N}(\mathrm{n})$ and the denominator $\mathrm{D}(\mathrm{n})$ are calculated. The power $\mathrm{P}(\mathrm{n})$ is calculated as the averaged zero-lag autocorrelation of the signal. $\mathrm{N}(\mathrm{n})$ and $\mathrm{D}(\mathrm{n})$ are calculated as the real and imaginary components of the single-lag auto-correlation. The two are used later to estimate the mean frequency of the signal which is calculated as the arctangent of the ratio of $\mathrm{N}(\mathrm{n}) / \mathrm{D}(\mathrm{n})$. 
The generated triplet vectors $\mathrm{P}(\mathrm{n}), \mathrm{N}(\mathrm{n})$ and $\mathrm{D}(\mathrm{n})$ are then filtered with an equiweight FIR filter with up to sixteen taps. This step is called adaptive axial filtering. It is used to provide for spatial smoothing along the flow direction. The size of the FIR filter may be adaptively changed in the depth direction as a function of an estimate calculated by filtering the power signal using samples eight positions ahead of the ones being used by the equi-weight FIR filter. The goal is to increase the signal-tonoise ratio by increasing the smoothing in the axial direction as the flow signal amplitude decreases. The calculated estimate is used to look-up the proper filter coefficients from a pre-computed table. The adaptive filter may also be used to decimate the size of the vectors to the required upper limit of 512 samples (to satisfy scan conversion flow requirements) when needed.

Once the adaptive axial filter function is finished a table look-up step is performed to reduce the dynamic range of the data for use with later processing steps. In addition, the $N(n)$ and $D(n)$ vectors are transformed from the Cartesian axis to the polar one generating the angle and magnitude vectors $\Phi(\mathrm{n})$ and $\mathrm{R}(\mathrm{n})$ respectively. The next step, key hole filter, is used to remove (set to zero) the values of $\Phi(n), R(n)$ and $\mathrm{P}(\mathrm{n})$ where the noise due to weak signals has corrupted the measured Doppler shift. This filter is implemented as a look-up table. The values of $\Phi(n)$ and $R(n)$ are used to read an on-off bit that is used either to pass the values of $\Phi(n), R(n)$ and $P(n)$ unchanged to the next stage (if on) or set to zero if (off).

Once the $\Phi(n), R(n)$ and $P(n)$ vectors are key hole filtered a $3 \times 3$ median filter is used to smooth the discontinuities in the flow data. The sorting step in the median filter is done based on the phase data. The median filter step is followed by the persistence calculation. In the persistence step the phase $\Phi(n)$, magnitude $R(n)$ and power $\mathrm{P}(\mathrm{n})$ are temporally filtered using an IIR filter acting as a decaying peak hold filter. The phase values from the current frame are compared with the phase values from the previous frame multiplied by a decay constant. If the phase value from the current frame is higher than the weighted one from the previous frame the current phase, magnitude and power are passed to the next stage. Otherwise the old values from the previous frame are passed to the next stage instead. Finally, the phase and magnitude vectors are converted back into the rectangular coordinates (back to $N(n)$ and $\mathrm{D}(\mathrm{n}))$. In addition, the $\mathrm{P}(\mathrm{n}), \mathrm{N}(\mathrm{n})$ and $\mathrm{D}(\mathrm{n})$ values are compressed further using an additional table look-up step.

\section{Mapping Methodology}

The idea behind the method described here is to divide the input frame into segments as close in size as possible then distribute them among the processing elements of the array to balance the computational load among all processors [4][5][6]. A graphical illustration of the load balancing scheme used to allocate the B-Mode, and Doppler frames among processors for both $1 \mathrm{D}$ and 2D arrays is shown in figures 2 and 3 below.

Once each processor gets its data segment the processing steps described in the previous section can be started. Since these processing steps are now done in parallel, additional communication steps to transfer the vectors located at the boundaries of 
each processor's data segment to its neighbors are needed. In the remainder of this section we will describe the parallel implementation of the processing steps outlined in the previous section along with the data transfers needed at each step. However, we will concentrate only on the steps that require communication of data between processors.
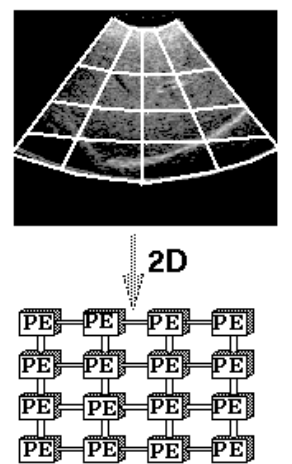

Fig. 2. Load balancing scheme for B-Mode processing for $1 \mathrm{D}$ and $2 \mathrm{D}$ arrays

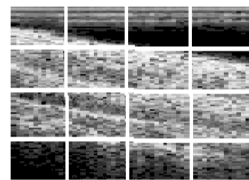

2D

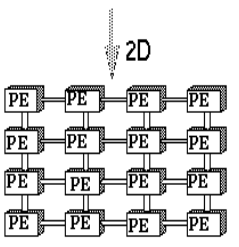

Fig. 3. Load balancing scheme for Doppler processing for $1 \mathrm{D}$ and $2 \mathrm{D}$ arrays

\subsection{B-Mode Processing}

In this section we will outline how the various processing steps were mapped onto the $1 \mathrm{D}$ and $2 \mathrm{D}$ architectures.

The magnitude calculation step is local in our mapping approach. The load balancing procedure gives each processor an even number of I and Q vectors. No data transfer between processors is, therefore, needed for this step.

In the focal zone blending step each processor performs the focal zone blending function on its local vectors. At the end of this step each processor may have partially blended vectors. This is because the vectors needed to blend some zones together may reside on different (adjacent) processors. In such cases some data transfer steps are needed to complete the blending. Two data communication steps (east and west) are used to transfer the partially blended vectors to the processor having the smallest number of overall local vectors [5]. A vector addition step is then used to finish the zone blending step. In the worst case each processor, except for the ones on the boundary of the array, could transfer up to two full vectors (one east and one west) in the case of 1D arrays. In the case of 2D arrays the size of the vectors transferred by each processor are now divided by the size of the processor array in the vertical direction (assuming equal distribution).

To perform axial edge enhancement a data transfer step along the axial (vertical) direction is needed so that local filtering of samples at the boundaries can be done. If the number of filter taps is $\mathrm{M}$, each processor except the ones at the boundary of the array needs (M-1) data samples sent to it from its north and south neighbors respectively. The two communication steps are needed only for the case of $2 \mathrm{D}$ arrays. In the case of $1 \mathrm{D}$ arrays each processor holds full vectors and therefore no south / north 
communication is needed. Once the north / south communication steps are finished all needed data is local and the filtering step is done locally on each processor.

The parallel frequency compounding function is performed in a way similar to that of the focal zone blending function which has been discussed earlier. Essentially, each processor adds together all vectors assigned to it which were down converted from the same RF vector. Some vectors belonging to the same RF vectors may be split among neighboring processors. For such cases the partially summed vectors may need to be transferred to the appropriate east / west neighbor following the same criteria used in the focal zone blending. The number of vectors transferred is also the same as that for the focal zone blending case. A final vector addition step is then used to add together the partially compounded vectors which now reside at the same node for each processor in the array.

The dynamic reject function also needs data transfer steps between neighboring processors along the vertical direction at the start of the computation. Again these data transfer steps are only needed for the case of $2 \mathrm{D}$ arrays. If $\mathrm{L}$ is the neighborhood window length and $\mathrm{M}$ is the guard band length, the number of samples transferred by each non boundary processor to its north and south neighbors is ( $\mathrm{L}-\mathrm{M}) / 2$. Once the north-south communication steps are done the dynamic reject function is performed by all processors in parallel with each processor operating on its local data.

To do the lateral edge enhancement in parallel we need to transfer data along the horizontal direction. Each processor, except the boundary ones, performs two data communication steps to their neighbors: east and west. For a three tap FIR filter based enhancement each processor would transfer two full vectors to its east and west neighbors respectively in the case of 1D arrays. In the case of $2 \mathrm{D}$ arrays the number of vectors transferred by each processor is still the same, the size of each transferred vector is now divided by the size of the processing array in the vertical direction, however.

The black hole filing step data transfer requirements for the parallel implementation are exactly the same as those of the lateral edge enhancement step. That is, each processor transfers one full vector to its east and west neighbors in the case of 1D. In the case of $2 \mathrm{D}$ the size of the vectors to be transferred is divided by the dimension of the array in the vertical direction.

\subsection{Doppler Processing}

In the current implementation the load balancing method used for flow processing assigns each processor complete sets of ensembles. With this data assignment, the adaptive wall filtering step becomes local to each processor with no data transfers.

The auto-correlation step is also performed on locally available full data ensembles. No data transfer is thus needed.

In the axial edge enhancement step the power, numerator and denominator vectors are filtered in the axial direction using an FIR filter (the current implementation uses a fixed kernel of length 16). To perform filtering in the axial direction a data transfer step along the axial (vertical) direction is needed to allow for the local filtering of samples at the boundaries. If the number of filter taps is 16 , each processor except the ones at the boundary of the array needs 15 samples sent to it from its north and 
south neighbors respectively. The two communication steps are needed only for the case of $2 \mathrm{D}$ arrays. In the case of $1 \mathrm{D}$ arrays each processor holds full vectors and no data transfer is needed.

The key hole filter function is implemented as a table look-up. No data transfer between processors is needed for this step.

To perform the median filter step on the vector triplets $(\Phi, \mathrm{R}, \mathrm{P})$ data transfers both in the axial and lateral directions are needed. Since the filter uses a $3 \times 3$ window, each non-boundary processor needs three data samples $(\Phi, R, P)$ from all of its neighboring processors (east, west, north and south) in the $2 \mathrm{D}$ array case. In the case of a $1 \mathrm{D}$ array each non boundary processor needs the three data samples $(\Phi, \mathrm{R}, \mathrm{P})$ from its east and west neighbors. Once all the data needed is transferred each processor performs the median filtering step locally.

The persistence step does not require any data communication. The needed data from a previous frame is stored locally on each processing node.

\section{SES/Workbench Simulation Model}

To verify our mapping approach and evaluate the performance of the mapped algorithms on the parallel architectures we built a high level simulation model using the SES / Workench tool [7]. The block diagram of the implemented 2D model is shown in figure 4. The SES/Workbench implementation of the model has different hierarchy levels.

At each level the functionality of one of the model blocks is refined. At the lowest levels the function to be performed is specified in C. The topology implemented in the model is dynamically reconfigurable to allow for the implementation of both 1D and 2D topologies of various sizes. Timing models of different DSP processors were developed and attached to the functions to get accurate timing information.

\section{$5 \quad$ Results}

Using the SES/Workbench model described in the previous section we performed the simulation using post digital receivers B-Mode, and Doppler frames as input. The goal was to verify the functionality of the approach and determine the performance characteristics of the parallelized algorithms on the two target architectures. In addition we wanted to determine the scalability of the mapped algorithms on the two architectures [8][9][10][11].

The processed frames using the parallel approach were collected from the local memory of each processor at the end of each run then displayed. Figures 5, and 6 show examples of a processed B-Mode, and Doppler frame. The B-Mode frame represents a liver example while the Doppler one represents a carotid artery. A few select output frames from the parallel implementation were also compared on a pixel by pixel basis with the results obtained from the original (serial) approach to validate the parallel approach.

The $C$ code used in the SES/Workbench model was also compiled using the cross compilers of several candidate DSPs. Timing models were then generated to estimate 
the performance of the system using such processors. The performance curves (scaleability) for TI's TMS320C40 DSP [12] are shown in figures 7 and 8.

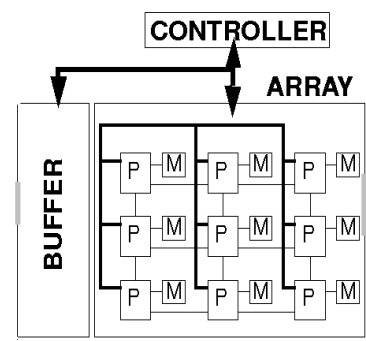

Fig. 4. SES Simulation model.

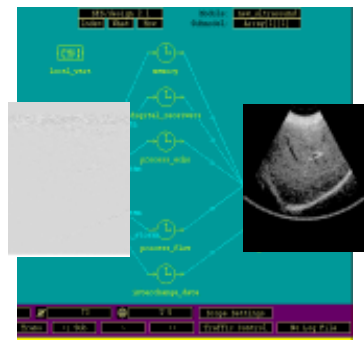

Fig. 5. A parallel based B- Fig. 6. A parallel based Doppler Mode liver frame

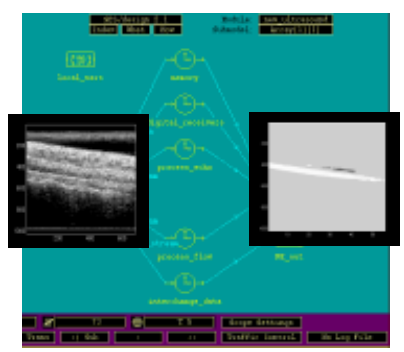

carotid artery frame

The performance metrics generated, namely the speed-up curves, indicate that the approach is scaleable for a high number of processors. This means that a machine based on such an architecture could be scaled to meet the performance requirements of market segments ranging from the low to high end. The cost projections for such a machine using the number of processors estimated from the simulation indicate that such an approach is also cost efficient. In our parallel approach, as the graphs show, the Doppler mode gives higher speed-up factors than the B-Mode. This is a reflection that in our parallel mapping / implementation the communication overhead as a function of the number of processors is lower for Doppler than that B-Mode in the range of interest. Both speed-up curves are linear for a fairly wide range, though.

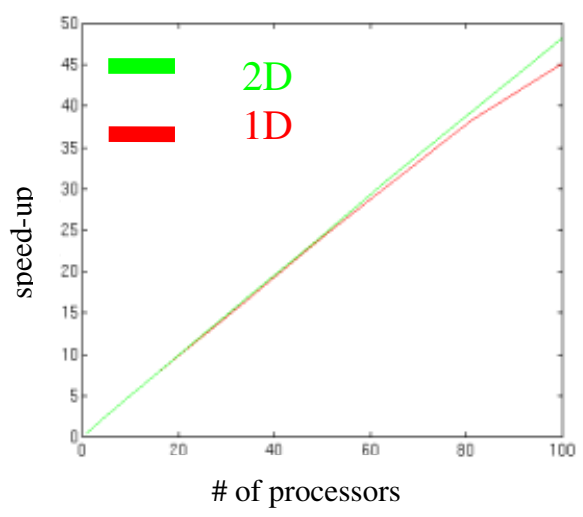

Fig. 7. Scaleability of B-Mode algorithms on $1 \& 2 \mathrm{D}$ arrays.

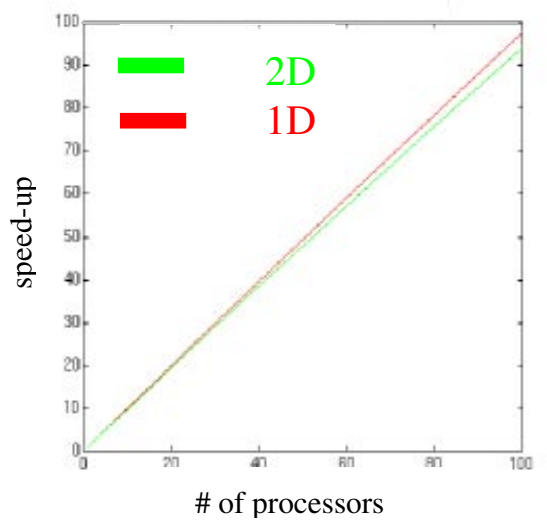

Fig. 8. Scaleability of Doppler algorithms on $1 \& 2 \mathrm{D}$ arrays.

\section{Conclusions}

In this paper, we have presented an overview of the ultrasound echo and flow processing pipelines. We also presented the parallelisation / mapping of the algorithms used in the two pipelines onto 1D and 2D processing arrays based architectures. The 
goal was to determine the scaleability of the parallel version of the algorithms on the two target architectures. We then build a high level simulation model to validate the approach. The results of the simulation verify the correctness and feasibility of the approach. The functional correctness of the approach was verified by comparing the frames processed using the parallel approach with those obtained from the uniprocessor implementation. The scaleability of the approach was determined to be linear in the number of processors for a wide range.

\section{References}

1. J. A. Jensen, Estimation of Blood Velocities Using Ultrasound: A signal Processing Approach, Cambridge University Press, Jan. 1996.

2. J. A. Zagzebski, Essentials of Ultrasound Physics, Mosby-Year Book, Jan. 1996.

3. L. R. Tarbox, "Description of Processing Algorithms in Ultrasound Scanners", Internal Report, SCR, Sept. 1993.

4. K. Hwang, "Advanced Computer Architectures: Parallelism, Scalablity, Programmability," McGraw Hill, 1993.

5. F. T. Leighton, "Introduction to Parallel Algorithms and Architectures: Arrays, Trees, Hypercubes," Morgan Kaufmann, San Mateo, CA 1992.

6. S. Y. Kung, "VLSI Array Processors," Prentice Hall, Englewood Cliffs, NJ, 1988.

7. SES Inc., SES/Workbench User's Manual, Release 2.1, Scientific and Engineering Software, Inc., 1992.

8. Nussbaum and A. Agarwal, "Scalability of Parallel Machines," Communications of the ACM, no. 3, vol. 34, pp. 57-61, 1991.

9. V. Kumar and A. Gupta, "Analyzing Scalability of Parallel Algorithms and Architectures," TR 91-18, Department of Computer Science, University of Minnesota, Minneapolis, MN 55455, June 1991.

10. M. D. Hill, "What is Scalability?," Scalable Shared Memory Multiprocessors, M. Dubois and S. S. Thakkar (eds.), Kluwer Academic Publishers, 1991.

11. J.R. Zorbas, D.I. Rebele and R.E. VanKooten, "Measuring the Scalability of Parallel Computer Systems," Proceedings of SuperComputing 89, pp. 832-841, 1989.

12. J.R. Zorbas, D.I. Rebele and R.E. VanKooten, Parallel Processing With the TMS320C40, Texas Instruments, 1994. 